\title{
The role of NF-кB-mediated JNK pathway in cognitive impairment in a rat model of sleep apnea
}

\author{
Fan Liu, Ting-Wei Liu, Jian Kang \\ Department of Respiratory and Critical Care Medicine, The First Hospital of China Medical University, Shenyang 110001, China \\ Contributions: (I) Conception and design: F Liu; (II) Administrative support: J Kang; (III) Provision of study materials or patients: F Liu; (IV) \\ Collection and assembly of data: J Kang; (V) Data analysis and interpretation: TW Liu; (VI) Manuscript writing: All authors; (VII) Final approval of \\ manuscript: All authors. \\ Correspondence to: Dr. Jian Kang. Department of Respiratory and Critical Care Medicine, The First Hospital of China Medical University, No. 155, \\ Nanjingbei Street, Heping District, Shenyang 110001, China. Email: kangjian58@163.com.
}

Background: The aim of this study is to determine the role of nuclear factor kappa B (NF- $\kappa \mathrm{B})$-mediated c-Jun N-terminal kinase (JNK) pathway in cognitive impairment induced by chronic intermittent hypoxia (CIH).

Methods: Ninety-six male Sprague-Dawley rats were randomly divided into 8 groups: sham group, sustained hypoxia $(\mathrm{SH})$ group, $\mathrm{CIH}$ group, $\mathrm{CIH}+$ melatonin group, $\mathrm{CIH}+$ vitamin $\mathrm{E}$ group, $\mathrm{CIH}+\mathrm{DMSO}$ group, $\mathrm{CIH}+\mathrm{BAY}$ 11-7082 group and $\mathrm{CIH}+$ normal saline (NS) group. Rats were exposed to normoxia, $\mathrm{CIH}\left(21 \% \mathrm{O}_{2}\right.$ for $60 \mathrm{~s}$ and $10 \% \mathrm{O}_{2}$ for $60 \mathrm{~s}$, cyclically repeated for $10 \mathrm{~h} /$ day $)$ or $\mathrm{SH}\left(10 \% \mathrm{O}_{2}\right.$ for $10 \mathrm{~h} /$ day $)$ for 14 days. Afterwards, Morris water maze test was conducted, and serum and hippocampus tissues were subjected to molecular biological and biochemical analyses.

Results: Compared with the Sham and SH group, oxidative stress was induced by CIH in rat hippocampus with the high level of malondialdehyde (MDA) and 8-iso-PGF2 $\alpha$ and the low level of superoxide dismutase (SOD) and glutathione (GSH). Activated NF- $\mathrm{BB}$ and its downstream products including tumor necrosis factor-alpha (TNF- $\alpha$ ), interleukin-6 (IL-6) and inducible nitric oxide synthase (iNOS) were highly expressed in $\mathrm{CIH}$ rats. These changes were attenuated by pretreatment of the rats with melatonin and vitamin $\mathrm{E}$. $\mathrm{CIH}$ also resulted in hippocampus neuron apoptosis with increased caspase 3 level, dUIP nick end labeling (TUNEL)-positive neurons number and cognitive impairment verified by prolonged latency and shortened time in the target quadrant in Morris water maze test. JNK and its downstream transcriptional factors including c-Jun, activating transcription factor 2 (ATF2), and JunD were all significantly phosphorylated in $\mathrm{CIH}$ rats. However, pretreatment of NF- $\kappa$ B inhibitor BAY 11-7082 inhibited the activation of NF- $\kappa$ B under $\mathrm{CIH}$ condition and also significantly reduced the phosphorylation of JNK as well as c-Jun, ATF2, and JunD. Moreover, hippocampus neuron apoptosis and cognitive impairment were significantly improved with the pretreatment of BAY 11-7082 in rats subjected to CIH.

Conclusions: These findings suggest that NF-кB-mediated JNK pathway is at least partially implicated in $\mathrm{CIH}$-induced hippocampus neuron apoptosis and cognitive impairment. Inhibition of NF- $\mathrm{B}$ activation provided a therapeutic potential for cognitive impairment in sleep apnea (SA).

Keywords: Chronic intermittent hypoxia (CIH); nuclear factor kappa B (NF- $\mathrm{B})$; c-Jun N-terminal kinase (JNK); melatonin; vitamin E; BAY 11-7082

Submitted Oct 15, 2018. Accepted for publication Dec 03, 2018.

doi: $10.21037 /$ jtd.2018.12.05

View this article at: http://dx.doi.org/10.21037/jtd.2018.12.05 


\section{Introduction}

Sleep apnea (SA), as a common chronic disease, is characterized by repeated episodes of full or partial obstruction of the upper respiratory tract during sleep (1). Recurrent airflow obstruction during sleep leads to chronic intermittent hypoxia $(\mathrm{CIH})$, which is a major hallmark in SA (2). Plenty of studies suggest that CIH-induced pathogenesis mainly includes oxidative stress, inflammation and cognitive deficits $(3,4)$. It is estimated that up to $30 \%$ of patients with SA have cognitive impairment (5). Additionally, the hippocampus is closely related to spatial learning and memorizing, and its injury is thought to be the major cause of cognitive impairment in SA patients $(6,7)$.

$\mathrm{CIH}$ is similar to repetitive ischemia/reperfusion, which leads to a large number of free radicals and different degrees of oxidative stress (8). Several studies revealed that oxidative stress from $\mathrm{CIH}$ could result in the damage of various organs $(9,10)$. The hippocampus was very vulnerable to oxidative stress and Yürüker et al. observed that oxidative stress was involved with the apoptosis of hippocampus neurons (11). It is generally accepted that the c-Jun N-terminal kinase (JNK) signaling pathway was closely associated with apoptosis (12). Multiple JNK apoptotic cascade elements such as Jun, ATF2 and JunD are all key factors in apoptotic process (13). However, it remains unknown whether the signaling molecules in the JNK pathway are involved in hippocampus damage in SA. In addition, accumulating evidence suggests that nuclear factor kappa $\mathrm{B}(\mathrm{NF}-\mathrm{\kappa B})$ has complex interaction with the JNK pathway and also regulates apoptosis in many cell types $(14,15)$. NF- $\mathrm{KB}$ in hippocampal neurons can be activated by reactive oxygen species (ROS) caused by intermittent hypoxia $(15,16)$. Thus, we hypothesized that NF- $\mathrm{KB}$-mediated JNK pathway may be relevant with $\mathrm{CIH}$-induced hippocampus neuron apoptosis and cognitive impairment.

In this study, we examined the levels of oxidative stress, activation of NF- $\kappa \mathrm{B}$, JNK pathway signals, cognitive function and neuron apoptosis in the hippocampus of rats after 14-days exposure to normoxia, $\mathrm{CIH}$ and sustained hypoxia $(\mathrm{SH})$ to find out the exact relationship between NF- $\mathrm{BB}$, JNK pathway and cognition impairment of SA. According to the previous studies, melatonin and vitamin $\mathrm{E}$ are known to be the effective antioxidants, which have anti-inflammatory and neuroprotective effects (17-20). BAY 11-7082, an inhibitor of NF- $\kappa \mathrm{B}$, can suppress inflammatory activity and improve neurological recovery $(21,22)$. Hence, we selected melatonin, vitamin $\mathrm{E}$ and BAY 11-7082 as treatment groups.

\section{Methods}

Animals

All animal studies were approved by the Institutional Animal Care and Use Committee of the First Hospital of China Medical University. Ninety-six male SpragueDawley rats weighing $200 \pm 5 \mathrm{~g}$ (Chinese Academy of Medical Sciences) were randomly divided into 8 groups: sham group, CIH group, chronic SH group, CIH plus melatonin intervention $(\mathrm{CIH}+$ melatonin) group, $\mathrm{CIH}$ plus vitamin $\mathrm{E}$ intervention $(\mathrm{CIH}+$ vitamin $\mathrm{E})$ group, $\mathrm{CIH}$ plus BAY 11-7082 intervention (CIH + BAY 11-7082) group, $\mathrm{CIH}$ plus DMSO control (CIH + DMSO) group and $\mathrm{CIH}$ plus normal saline control $(\mathrm{CIH}+\mathrm{NS})$ group. There were 12 rats in each group. The standard food and drinking water were provided for all rats.

\section{Intermittent bypoxia and SH in rats}

Rats were placed in normobaric chambers, and all rats were free to move in the cabin and access to water and food freely. Oxycycler model A84XOV (Biospherix, Redfield, NY, USA) was used to achieve CIH and SH. The model of CIH was established according to previously published methods (10). The intermittent hypoxia mode was as follows: intra-cabin oxygen concentration of $21 \% \mathrm{O}_{2}$ for $60 \mathrm{~s}$ and $10 \% \mathrm{O}_{2}$ for $60 \mathrm{~s}$. The above cycle of intermittent hypoxia was maintained for 10 hours (8:00 AM to 6:00 PM) for 14 days. The SH mode was as follows: intracabin oxygen concentration was maintained at $10 \% \mathrm{O}_{2}$ for 10 hours (8:00 AM to 6:00 PM) for 14 days. Rats in the sham group were placed in an identical cabin (8:00 AM to 6:00 PM) with access to normal room air.

\section{Vitamin E, melatonin and BAY 11-7082 intervention}

Vitamin E (100 mg/kg, Sigma-Aldrich, St Louis, MO, USA) was injected into the rats in the $\mathrm{CIH}+$ vitamin E group via gastric tube 30 minutes before the intermittent hypoxia exposure. The rats in the $\mathrm{CIH}+$ melatonin group were injected with melatonin (Sigma-Aldrich, St Louis, MO, USA) intraperitoneally at a dose of $400 \mu \mathrm{g} / \mathrm{kg}$ for each day 30 minutes before the intermittent hypoxic exposure. BAY 11-7082 (Merck, Darmstadt, Germany) was first dissolved in a small amount of DMSO and then diluted by NS 
according to the previously published method (23). The rats in the CIH + BAY 11-7082 group were intraperitoneally injected at a dose of $20 \mathrm{mg} / \mathrm{kg} 30$ minutes before intermittent hypoxia exposure. The rats in the $\mathrm{CIH}+\mathrm{NS}$ group were injected with $0.5 \mathrm{~mL}$ of $\mathrm{NS}$ intraperitoneally 30 minutes before the intermittent hypoxia exposure. The rats in the $\mathrm{CIH}+\mathrm{DMSO}$ group were injected with $0.5 \mathrm{~mL}$ of $0.2 \%$ DMSO intraperitoneally 30 minutes before the intermittent hypoxia exposure.

\section{Tissue acquisition}

Rats were anaesthetized by intraperitoneal injection of $100 \mathrm{mg} / \mathrm{kg}$ sodium pentobarbital $(0.2 \mathrm{~mL} / 100 \mathrm{~g})$, and then the skull was rapidly dissected and the brain tissue was completely removed. The hippocampus could be seen by opening the brain tissue coronally approximately $3.5 \mathrm{~mm}$ after the optic chiasm. Then the hippocampus was isolated and stored at $-80{ }^{\circ} \mathrm{C}$ rapidly for further use. To prepare hippocampal tissue sections, tissues were fixed in $4 \%$ paraformaldehyde for more than 24 hours. Five consecutive sections of coronal were used for TUNEL staining.

\section{Determination of oxidative stress-related indicators}

The BCA protein assay kit (ThermoFisher Scientific, Shanghai, China) was used to quantify protein concentration. Malondialdehyde (MDA) and superoxide dismutase (SOD) levels were measured by the thiobarbituric acid (TAB) and xanthine oxidase method, respectively (Nanjing Jiancheng Bioengineering Institute). The level of 8 -iso-PGF2 $\alpha$ was determined using a doubleantibody sandwich ABC-ELISA (Cayman) based on the manufacturer's instruction. Glutathione (GSH) was measured using the GSH assay kit (Nanjing Jiancheng Bioengineering Institute) based on the manufacturer's instruction.

\section{Cytoplasmic and nuclear protein extraction}

Nuclear and cytoplasmic fractions were isolated using the P0028 kit of Beyotime Biotechnology based on the manufacture's instruction. The hippocampal tissue was cut into small pieces and treated by cytoplasm protein extraction reagents $\mathrm{A}$ and $\mathrm{B}$, and then PMSF was added to prepare a tissue homogenate with a final concentration of $1 \mathrm{mM}$. The tissue homogenate was homogenized and centrifuged to obtain the supernatant that contained some cytoplasmic proteins. After collecting the supernatant, the remained cell pellets were added to nuclear protein extraction reagent to collect the supernatant containing nuclear proteins. The purification was verified by immunoblotting with the nuclear nucleolin and cytosolic specific actin antibodies $(1: 1,000$, Abcam $)$ as described in the next section.

\section{Immunoblot analysis}

Protein quantification was performed using the Pierce BCA Protein Assay Kit, and $50 \mu \mathrm{g}$ of total protein was dissolved by polyacrylamide gel electrophoresis (SDS-PAGE, $8 \%$ ). Membranes were probed with anti-rat antibodies of P65 (1:2,500; Santa Cruz, sc-8008), JNK (1:1,000 dilution; Santa Cruz, sc-7345), p-JNK (1:200 dilution; Santa Cruz, sc-293136), c-jun (1:1,000 dilution; Santa Cruz, sc-166540), p-c-jun (1:200 dilution; Santa Cruz, sc-53182), p-JunD (1:500 dilution; Abcam, ab139180), JunD (1:500 dilution; Abcam, ab181615), p-ATF2 (1:1,000 dilution; Abcam, ab32019), ATF2 (1:500 dilution; Abcam, sab47476), actin (1:500; Santa Cruz, sc-58673) and Lamin A/C (1:500; Santa Cruz, sc-7293) at $4{ }^{\circ} \mathrm{C}$ overnight after blocking for $60 \mathrm{~min}$. The blots were imaged using ECL Plus western blotting detection reagents. The Image $\mathrm{J}$ software was used to determine the average absorbance value of the corresponding bands.

\section{Estimation of TNF- $\alpha, I L-6$ and iNOS expression level}

Total RNA from rat hippocampus tissue was extracted with Trizol regent (ThermoFisher Scientific). The mRNA levels were determined by qRT-PCR using the following primers-tumor necrosis factor-alpha (TNF- $\alpha$ ): forward5'-CTG TGC CTC AGC CTC TTC TCA TTC-3' and reverse-5'-TTG GGA ACT TCT CCT CCT TGT TGG3'; interleukin-6 (IL-6): forward-5'-ACA AGT CGG AGG CTT AAT TAC ACA T-3' and reverse-5'- AAT CAG AAT TGC CAT TGC ACA A-3'; inducible nitric oxide synthase (iNOS): 5'-CCT CCT CCA CCC TAC CAA GT-3' and 5'-CAC CCA AAG TGC TTC AGT CA-3'. The protein level of TNF- $\alpha$ was determined using the TNF- $\alpha$ ELISA kit according to the manufacturer's instruction (Yokosai Biotech Co., Ltd. Cat No. ER006).

\section{Determination of cognitive function}

Water maze test was carried out as described previously $(24,25)$. The water maze was a circular pool with a diameter 
of $130 \mathrm{~cm}$ and a height of $50 \mathrm{~cm}$. The water depth of the water maze was $30 \mathrm{~cm}$, and the water temperature was kept at $(25 \pm 2){ }^{\circ} \mathrm{C}$. The pool was divided into four quadrants (I, II, III, and IV). A circular platform (diameter: $9 \mathrm{~cm}$; height: $29 \mathrm{~cm}$ ) was placed under $1 \mathrm{~cm}$ of the surface of the water in the center of the quadrant I. A video camera with a display system was placed above the maze to record the tracks of rats synchronously. The navigation test lasted for 4 consecutive days. Four acquisition trials per day were performed. We observed and recorded required time (latency) of the rat to find and climb onto the platform. The spatial probe test was undergone $2 \mathrm{~h}$ after the last acquisition trial on the last day of training.

\section{Caspase-3 activity assay}

The activity of cysteine aspartyl-specific protease (caspase) 3 was measured in the hippocampus using the caspase 3 colorimetric viability assay kit (Promega, Promega Madison, Wisconsin, USA) based on manufacturer's instruction.

\section{In situ dUIP nick end labeling (TUNEL) assay}

Apoptosis was detected in situ using TUNEL mediated by oligonucleotide-end deoxyribonucleotidyltransferase (TdT) (Nanjing Jiancheng Bioengineering Institute) based on manufacturer's instructions. Slides were evaluated under light microscope: nucleus with blue dye was labeled as TUNEL "-", representing normal cells; nucleus with brown staining was labeled as TUNEL “+”, representing apoptotic cells. Five fields were randomly counted to calculate the apoptotic rate of hippocampal neurons.

\section{Statistical analysis}

All data were expressed as the mean \pm standard deviation (SD). Comparisons between groups were performed by a one-way analysis of variance (ANOVA). $\mathrm{P}<0.05$ was considered to indicate a statistically significant difference. Statistical analyses were performed with SPSS 17.0 software for Windows (IBM Corp., Armonk, NY, USA). All experiments were repeated at least 3 times.

\section{Results}

\section{Levels of oxidative stress in rat hippocampus}

$\mathrm{CIH}$ caused significant oxidative stress in rat hippocampus.
MDA and 8-iso-PGF2 $\alpha$ in hippocampus were significantly increased in the $\mathrm{CIH}$ group $(\mathrm{P}<0.01)$ (Figure $1 A, B)$, SOD and GSH level were decreased (Figure $1 C, D$ ). SH did not cause oxidative stress. Antioxidants of melatonin and vitamin $\mathrm{E}$ administrated to $\mathrm{CIH}$ rats significantly decreased the expression of MDA and 8-iso-PGF2 $\alpha$ and increased the expression of SOD and GSH $(\mathrm{P}<0.05)$ (Figure 1). NF- $\mathrm{kB}$ inhibitor BAY 11-7082 had no obvious effect on oxidative stress (Figure 1).

\section{Morris water maze test}

All rats had improved performance during acquisition according to the lowering in the escape latency over the 4 training days (Figure 2). On days 1 and 2, there was no significant difference in escape latency among all the groups, which meant all rats had similar motor and visual capabilities. Compared with the sham group, the escape latency of rats in the $\mathrm{CIH}$ group was significantly longer for days 3 and $4(\mathrm{P}<0.01)$ (Figure $2 A)$. We also found significant differences between the $\mathrm{CIH}$ and Sham groups with regard to staying time in the target quadrant $(\mathrm{P}<0.01)$ (Figure $2 B)$. Rats pre-treated with melatonin, vitamin $\mathrm{E}$, or BAY 117082 had significantly shorter escape latency for days 3 and 4 and longer staying time in the target quadrant compared with the $\mathrm{CIH}$ group $(\mathrm{P}<0.05)$ (Figure $2 A, B)$. No statistically significant difference was detectable between the sham and SH groups (Figure $2 A, B)$.

\section{Activation and inbibition of $\mathrm{NF}-\boldsymbol{\kappa} B$ and the $7 \mathrm{NK}$ pathway signals}

To evaluate the activation of NF- $\mathrm{NB}$ by $\mathrm{CIH}$, we first performed immunoblotting to verify the purification with the nuclear nucleolin and cytosolic specific actin (Figure $3 A, B$ ). Then, the immunoblot analysis indicated that both $\mathrm{CIH}$ and $\mathrm{SH}$ could promote the translocation of P65 into the nucleus in rat hippocampus compared with the sham group, and there was more significant difference in the CIH group (Figure 3C,D). In addition, the expression level of the phosphorylation of JNK and its downstream signaling molecules including c-Jun, ATF2, and JunD were also detected by immunoblotting assay in 8 groups, with the relatively high expression in the $\mathrm{CIH}, \mathrm{CIH}+\mathrm{NS}$ and $\mathrm{CIH}+\mathrm{DMSO}$ groups (Figure 3C,E,F,G,H). Vitamin E and melatonin partially inhibited the nuclear translocation of P65 induced by CIH, and the phosphorylation of JNK and its downstream signaling molecule were lower. BAY 11- 

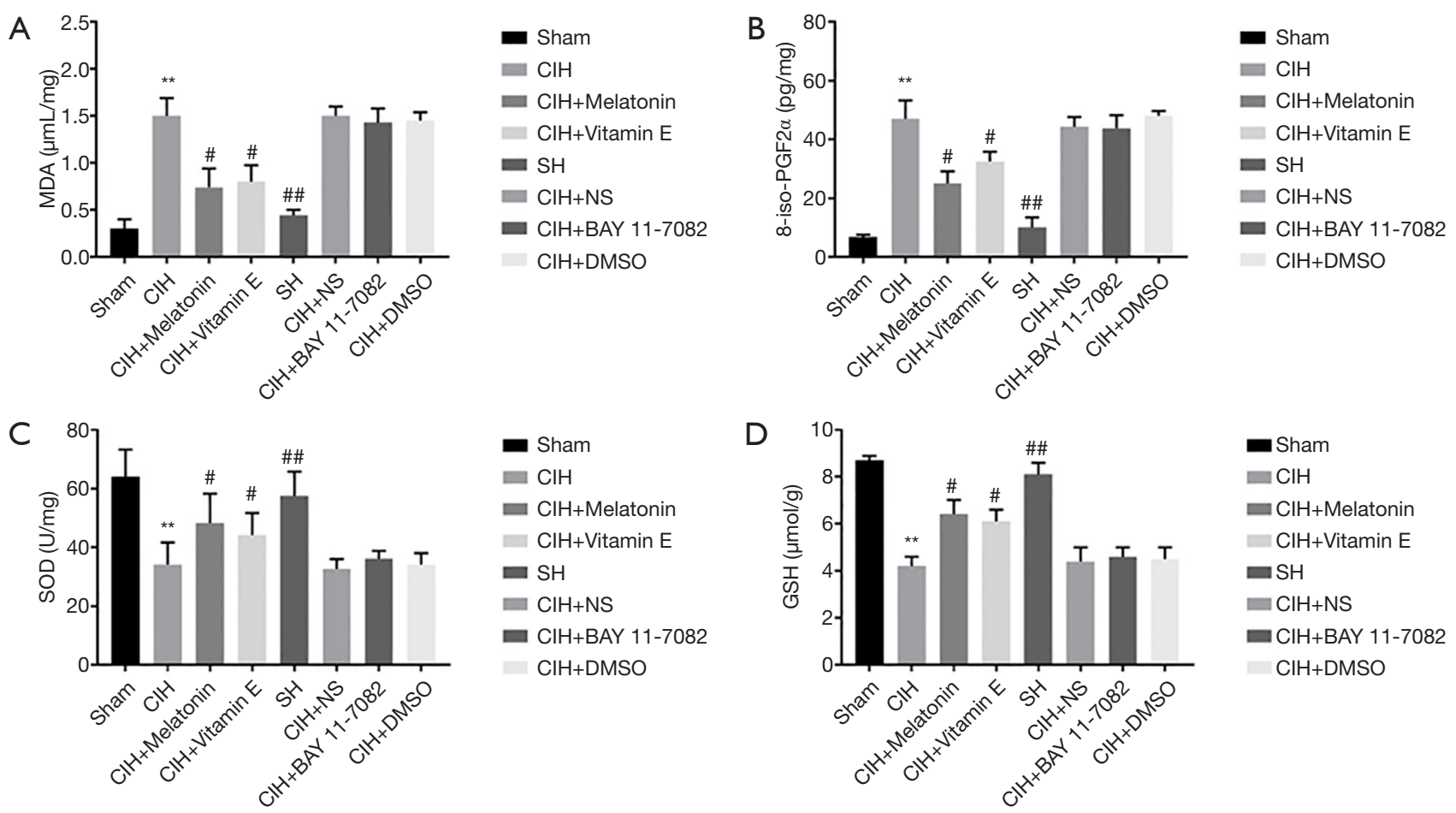

Figure 1 Levels of oxidative stress. (A,B) CIH increased MDA and 8-iso-PGF2 $\alpha$ levels in rat hippocampus. Pretreatment with melatonin and vitamin $\mathrm{E}$ attenuated these increments. NF- $\mathrm{kB}$ inhibitor BAY 11-7082 had no effect on these increments. (C,D) CIH decreased SOD and GSH. Pretreatment with melatonin and vitamin $\mathrm{E}$ attenuated these decrements. BAY 11-7082 had no effect. ${ }^{* *}, \mathrm{P}<0.01$ vs. sham group; \#, $\mathrm{P}<0.05$, ,, $\mathrm{P}<0.01$ vs. CIH group. Results representative of three independent experiments. CIH, chronic intermittent hypoxia; MDA, malondialdehyde; SOD, superoxide dismutase; GSH, glutathione; NS, normal saline; SH, sustained hypoxia.
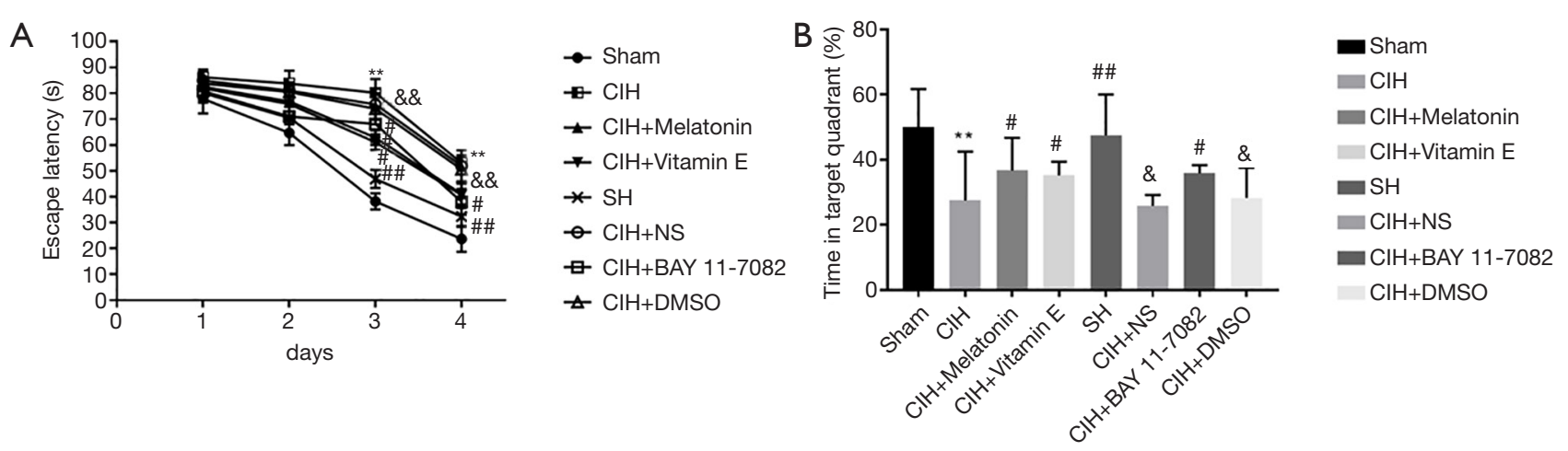

Figure 2 Changes in cognitive behavior. (A,B) CIH prolonged latency for days 3 and 4 and shortened time in target quadrant, which was attenuated by melatonin, vitamin $\mathrm{E}$ and $\mathrm{BAY} 11-7082 .{ }^{* *}, \mathrm{P}<0.01$ vs. sham group; ${ }^{*}, \mathrm{P}<0.05,{ }^{\text {\#\# }}, \mathrm{P}<0.01$ vs. $\mathrm{CIH}$ group; ${ }^{*}, \mathrm{P}<0.05$ vs. $\mathrm{CIH}$ + BAY 11-7082 group; ${ }^{\& \&}, \mathrm{P}<0.01$ vs. CIH + BAY 11-7082 group. Results representative of three independent experiments. CIH, chronic intermittent hypoxia; NS, normal saline; SH, sustained hypoxia. 
A
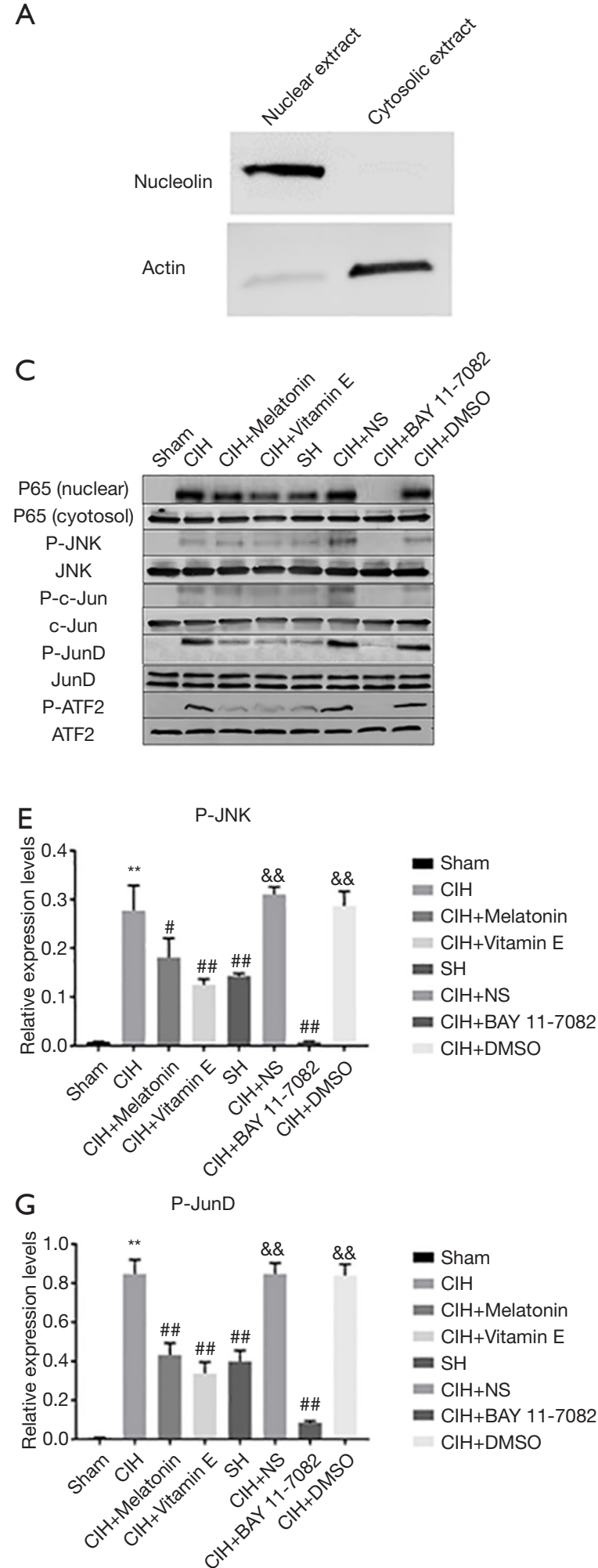

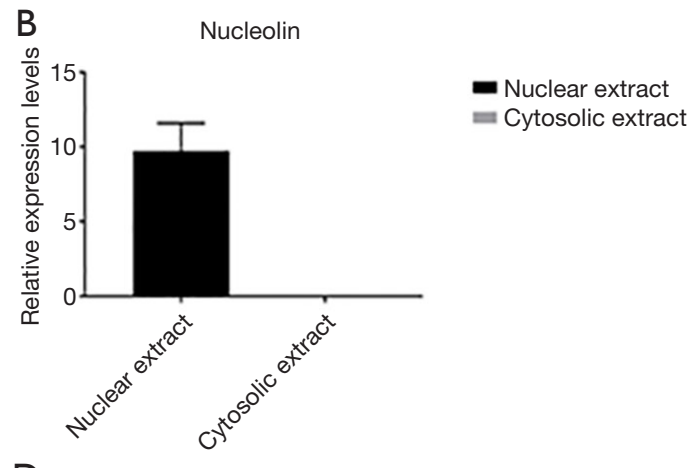

D
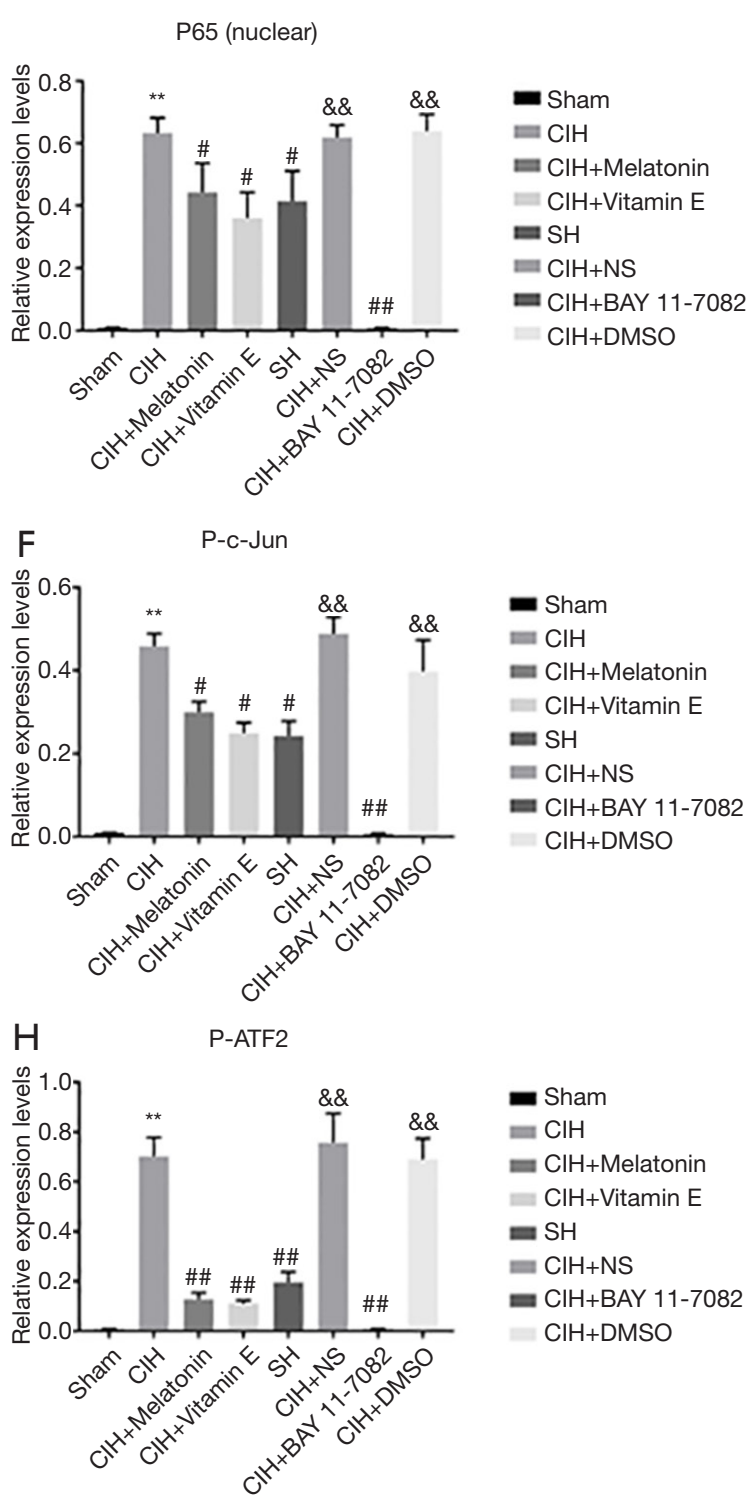

Figure 3 Activation and inhibition of NF-kB and JNK pathway signals. (A,B) Verification of purity of cytoplasmic and nuclear fractionation. (C,D,E,F,G,H) Immunoblot analysis of nuclear and cytoplasmic P65, P-JNK, total-JNK, P-c-Jun, c-Jun, ATF2, P-ATF2, JunD, and P-JunD. Results representative of three independent experiments. **, $\mathrm{P}<0.01$ vs. Sham group; ${ }^{\prime}, \mathrm{P}<0.05,{ }^{\text {\#\#, }}, \mathrm{P}<0.01$ vs. $\mathrm{CIH}$ group; ${ }^{\text {sck }}, \mathrm{P}<0.01$ vs. CIH + BAY 11-7082 group. NF-kB, nuclear factor kappa B; JNK, c-Jun N-terminal kinase; CIH, chronic intermittent hypoxia; NS, normal saline; SH, sustained hypoxia. 

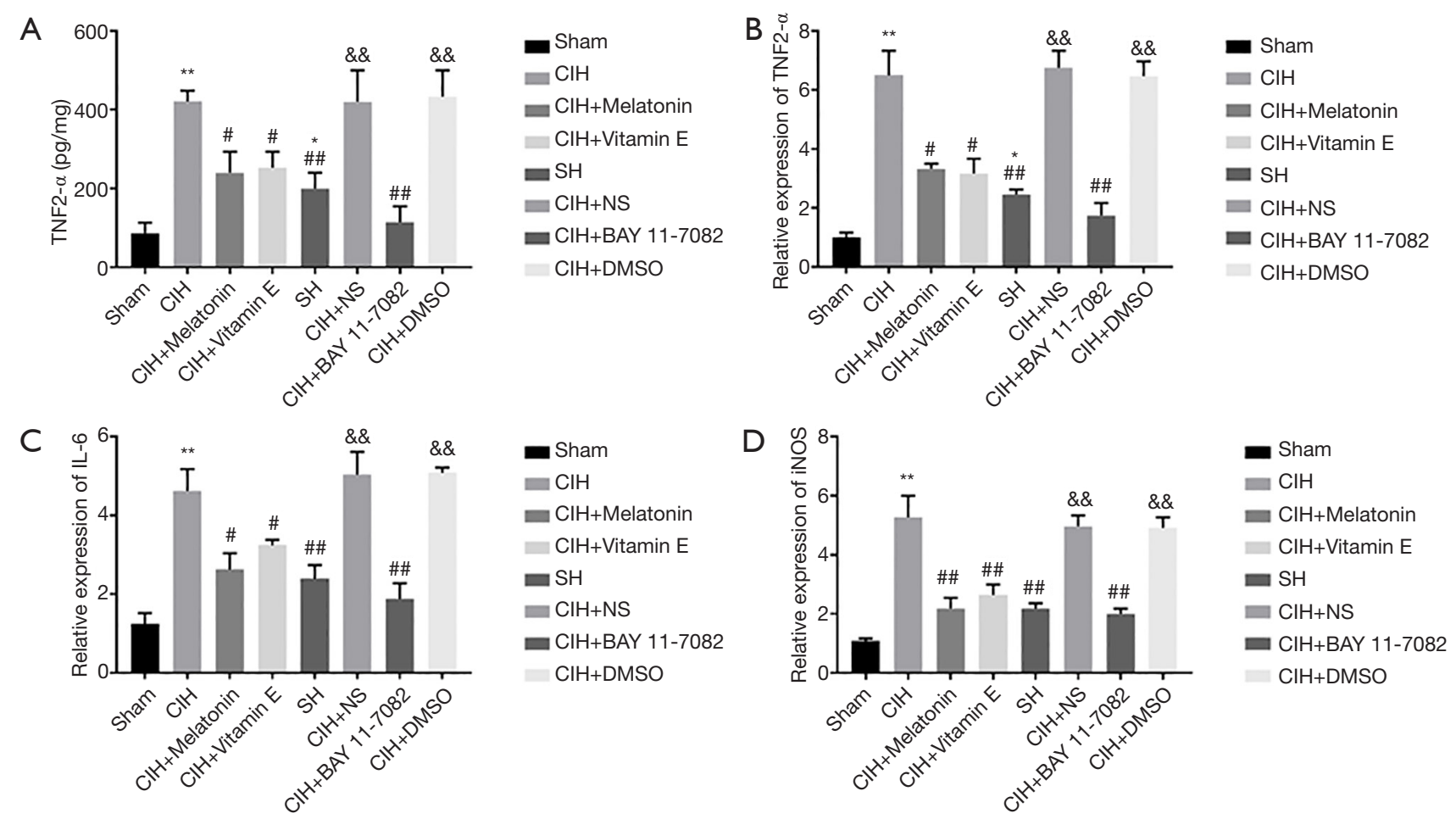

Figure 4 Expression of TNF $\alpha$, IL-6 and iNOS. (A,B) The expression of TNF $\alpha$ mRNA and protein, as well as the mRNA expression of IL6, and iNOS (C,D) in rat hippocampus in the indicated groups. * $\mathrm{P}<0.05,{ }^{* *}, \mathrm{P}<0.01$ vs. sham group; ${ }^{\#}, \mathrm{P}<0.05,{ }^{\# \#}, \mathrm{P}<0.01$ vs. CIH group; ${ }^{*} \&$, $\mathrm{P}<0.01$ vs. $\mathrm{CIH}+\mathrm{BAY} 11-7082$ group. Results representative of three independent experiments. CIH, chronic intermittent hypoxia; iNOS, inducible nitric oxide synthase; NS, normal saline; SH, sustained hypoxia.

7082 greatly reduced the migration of P65 into the nucleus under CIH condition and almost totally suppressed the phosphorylation of JNK and its downstream signaling molecules of c-Jun, ATF2, and JunD.

\section{Expression of TNFa, IL-6 and iNOS}

Consistent with the validated activation of NF- $\kappa \mathrm{B}$ by $\mathrm{CIH}$, the expression of TNF- $\alpha$ mRNA, TNF- $\alpha$ protein, IL-6, and iNOS was increased $(\mathrm{P}<0.01)$ (Figure 4). Antioxidants of either melatonin or vitamin $\mathrm{E}$ administration prior to $\mathrm{CIH}$ exposure significantly reduced these increments suggesting that the activation of $\mathrm{NF}-\mathrm{\kappa B}$ was induced by ROS from the oxidative stress $(\mathrm{P}<0.05)$. NF- $\mathrm{kB}$ inhibitor BAY 11-7082 reduced TNF- $\alpha$, IL-6, and iNOS expressions even more $(\mathrm{P}<0.01)$, and these molecules expression levels had no significant differences between the BAY 11-7082 treatment group and the sham group. SH caused the nuclear translocation of $\mathrm{P} 65$ as shown by immunoblot, and it also increased the expressions of TNF- $\alpha$ mRNA, TNF- $\alpha$ protein, IL-6, and iNOS $(\mathrm{P}<0.05)$, but these increments were significantly lower than those in the CIH group $(\mathrm{P}<0.01)$. Since there was no evidence of oxidative stress in $\mathrm{SH}$ group, this activation of NF- $\mathrm{\kappa B}$ by $\mathrm{SH}$ did not seem to be mediated by ROS.

\section{Apoptosis of hippocampus neurons}

We used caspase- 3 activity assay and TUNEL assay to detect the effect of $\mathrm{CIH}$ on the hippocampus neurons apoptosis. As shown in Figure 5, CIH caused apoptosis of hippocampus neurons as proved by the increased activity of caspase 3. SH did not induce apoptosis. Both vitamin $\mathrm{E}$ and melatonin inhibited the activity of caspase 3 induced by $\mathrm{CIH}$ and protected hippocampus neurons from apoptosis $(\mathrm{P}<0.05)$. BAY 11-7082 significantly reduced caspase 3 activity and protected hippocampus neurons from apoptosis. Moreover, TUNEL assay further confirmed the number of TUNEL-positive neurons tended to be relatively high in the CIH group, whereas the large number of TUNEL- 


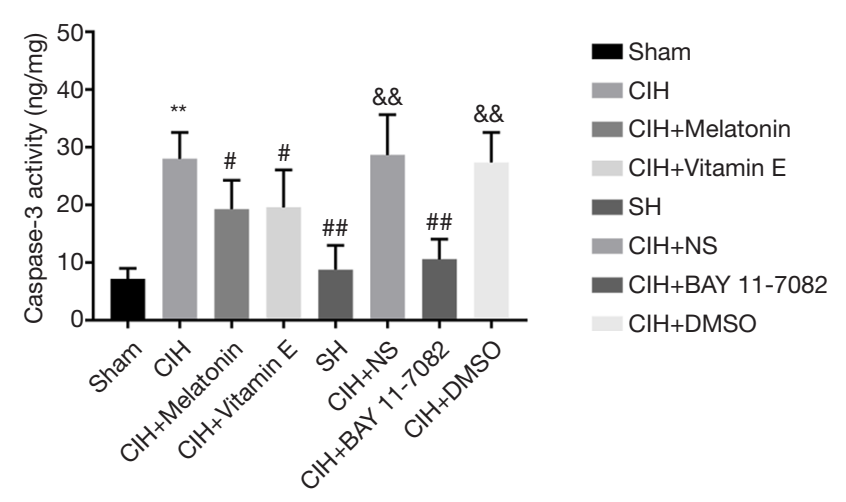

Figure 5 Caspase 3 activity in hippocampus in indicated groups. **, $\mathrm{P}<0.01$ vs. sham group; ${ }^{\#}, \mathrm{P}<0.05,{ }^{\# \#}, \mathrm{P}<0.01$ vs. CIH group; ${ }^{\text {\& } ~}$, $\mathrm{P}<0.01$ vs. $\mathrm{CIH}+\mathrm{BAY} 11-7082$ group. Results representative of three independent experiments. $\mathrm{CIH}$, chronic intermittent hypoxia; NS, normal saline; SH, sustained hypoxia.

positive neurons induced by $\mathrm{CIH}$ was respectively reduced by vitamin E, melatonin and BAY 11-7082. Particularly, there was a little number of TUNEL-positive neurons in the SH and BAY 11-7082 groups (Figure 6). Collectively, $\mathrm{CIH}$ obviously induced the apoptosis of hippocampus neurons.

\section{Discussion}

SA patients have periodic hypoxia-reoxygenation and frequently present with cognition impairment owing to hippocampus injury. However, the underlying molecular mechanisms for cognition impairment in SA are unclear. The current study established a rat model of SA by CIH challenge. We found that $\mathrm{CIH}$ caused intense oxidative stress and neuron apoptosis in rat hippocampus and resulted in impairment of learning and memorizing ability of rats. Oxidative stress effectively activated NF- $\mathrm{kB}$ and JNK and its downstream signaling molecules, which could be suppressed by pretreatment of the NF- $\mathrm{BB}$ inhibitor BAY 11-7082. BAY 11-7082 also attenuated the neuron apoptosis in the hippocampus and impairment of learning and memorizing ability of rats subjected to $\mathrm{CIH}$.

Repetitive breathing cessation and/or reduction of airflow due to partial or complete obstruction of the upper respiratory airways in SA patients finally lead to CIH. This CIH can produce a large number of ROS and cause oxidative damages $(9,10)$. Our study showed that CIH caused significant oxidative stress in the rat hippocampus, and the stress could be partially ameliorated by pretreated antioxidants of either vitamin $\mathrm{E}$ or melatonin. In agreement with the previous reports, vitamin $\mathrm{E}$ or melatonin could improve the impairments of oxidative damages in various diseases $(26,27)$. Thus, we established a rat model of SA with oxidative stress from CIH that could be the fundamental pathophysiological changes of SA.

Hippocampus injury is frequently found in SA patients and is thought to be the major cause of cognitive impairment $(6,7)$. Varying degrees of cognitive impairment and hippocampus neuron apoptosis in rats are observed under CIH (28-30). The present study revealed impaired learning and memorizing ability, increased activity of caspases 3 and in situ apoptosis of hippocampus neurons in the rat model of SA, but not in rats with SH exposure. $\mathrm{NF}-\kappa \mathrm{B}$ modulates gene expression in diverse cellular processes including apoptosis. Oxidative stress can activate $\mathrm{NF}-\kappa \mathrm{B}$ and increase the expression of proinflammatory cytokine of TNF- $\alpha$ (31). A previous study showed that NF- $\kappa$ B inhibitor BAY 11-7082 reduced the expression of TNF- $\alpha$ and attenuated apoptosis in a rat cardiac ischemiareperfusion injury model (32). In the present study, our results confirmed that BAY 11-7082 in SA rat model could inhibit the activation of NF- $\kappa \mathrm{B}$, reduce the expression of TNF- $\alpha$ in hippocampus and attenuate neuron apoptosis. JNK, also known as stress-activated protein kinases, is a member of the MAPK family. It is shown that JNK activation triggers myocardial cell apoptosis and exaggerate myocardial injury following ischemia/reperfusion (33). Inhibiting the ischemia/reperfusion-induced activation of JNK can reduce cardiomyocyte apoptosis (34). NF- $\mathrm{KB}$ is known to have subtle interaction with JNK; the inhibition of activation of NF- $\kappa \mathrm{B}$ is associated with attenuated the phosphorylation of JNK (14). The present study detected intense NF- $\mathrm{\kappa B}$ activation and JNK activation together with hippocampus neuron apoptosis and cognitive impairment after 14-days intermittent hypoxia exposure, and they were all significantly attenuated by the pretreatment of NF- $\mathrm{KB}$ inhibitor BAY 11-7082, which suggested JNK might induce lesions in the hippocampus tissue and cognitive dysfunction of rats subjected to $\mathrm{CIH}$ in an NF- $\mathrm{KB}$-dependent fashion.

In order to further understand the mechanism of BAY 11-7082 on the apoptosis of the hippocampus neuron, we studied the transcriptional factors of c-Jun, JunD and ATF2, the substrates of JNK involved in cell apoptosis. JNK regulates the activity of these apoptotic cascade elements by phosphorylation. The importance of the c-Jun protein in apoptosis induction is substantiated by previous studies (35). 

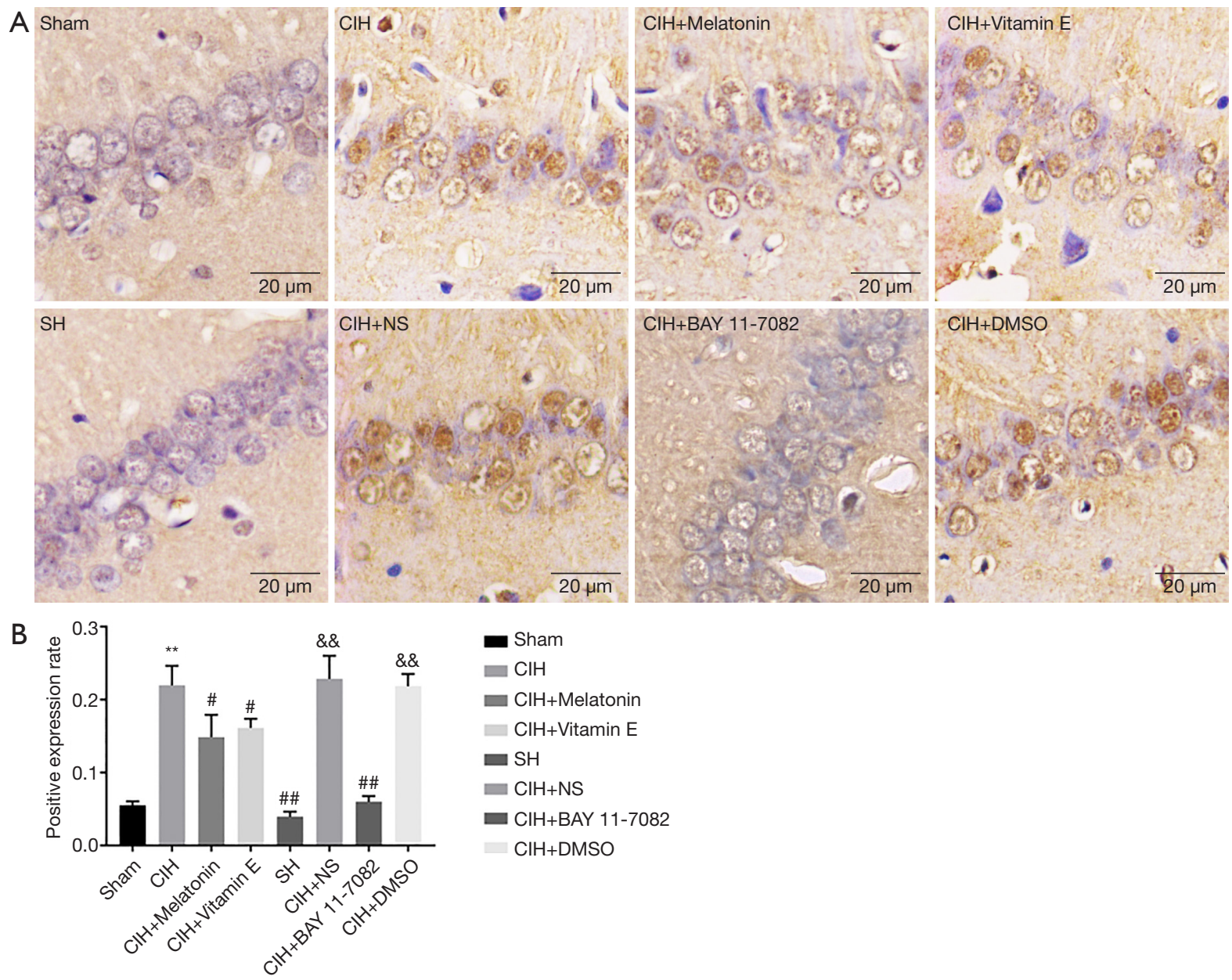

Figure 6 The effect of CIH on the hippocampus neurons apoptosis. (A) TUNEL technique in situ detection of apoptosis of hippocampal neurons in indicated groups. (B) Quantitative analysis of the positive expression rate was performed by Image Pro Plus software (version 6.0). Brown nuclear stain indicates TUNEL “+”. Results are representative of 3 different animals in each group. **, $\mathrm{P}<0.01$ vs. sham group; \#, $\mathrm{P}<0.05$, \#, $\mathrm{P}<0.01$ vs. $\mathrm{CIH}$ group; ${ }^{\& \&}, \mathrm{P}<0.01$ vs. $\mathrm{CIH}+\mathrm{BAY} 11-7082$ group. $\mathrm{CIH}$, chronic intermittent hypoxia; NS, normal saline; SH, sustained hypoxia.

JunD is a component of the activator protein-1 transcription factor complex, which is critical for cell survival (12). JunD induces apoptosis with regulation of proteins GADD45 $\alpha$ and GADD45 $\gamma$ (36), and represses Bcl-xL, a member of the Bcl-2 family of cell death suppressors, suggesting a complex link between apoptosis and JunD (37). Increased phosphorylation of ATF2 is associated with the chondrocyte apoptosis in Kashin-Beck disease (38). Activation of ATF-2 affects a series of target genes involved in cell apoptosis (39). In this study, we found that the phosphorylation of c-Jun, JunD and ATF-2 were all significantly reduced together with the attenuated phosphorylation of JNK and hippocampus neuron apoptosis and improved cognitive function after NF- $\kappa B$ inhibition. These findings further confirmed the implication of NF- $\mathrm{KB}$-mediated JNK pathway in hippocampus injury and cognitive dysfunction of SA rat model.

Continuous positive airway pressure (CPAP) is a wellestablished gold-standard treatment for SA, but it has limited effect on the cognitive performance, especially in those patients with poor compliance (40). There is an urgent need for pharmacological intervention in this medical situation. Our study suggested inhibiting the activation of NF- $\mathrm{KB}$ might represent a potentially important therapeutic approach cognitive impairment in SA. Of course, our study has limitations that SA patients have 
much longer (up to several years or even decades) exposure to intermittent hypoxia than the animals in this study. The degree and reoxygenation process are also more diverse and complex. The rat model of SA in our study only partially mimicked the pathophysiological changes of SA patients. It is generally considered that the function of signaling molecules including NF- $\mathrm{KB}$, JNK and its downstream cascadic elements depend on many factors such as cell type, nature of the stimulus, duration of its activation, activity of other signaling pathways and so on. More studies might be needed to further investigate NF- $\mathrm{KB}$ and the JNK pathway molecules in various experimental $\mathrm{CIH}$ settings.

In conclusion, caspase mediated pulmonary neurons apoptosis was responsible for cognitive dysfunction in $\mathrm{SA}$ and was regulated by NF- $\mathrm{KB}$ through JNK signaling pathway with the involvement of c-Jun, JunD and ATF-2. $\mathrm{NF}-\mathrm{\kappa B}$ Inhibitor might be a potential therapeutic agent for cognitive impairment in SA in the future.

\section{Acknowledgements}

None.

\section{Footnote}

Conflicts of Interest: The authors have no conflicts of interest to declare.

Ethical Statement: All animal studies were approved by the Institutional Animal Care and Use Committee of the First Hospital of China Medical University (No. KT2016046).

\section{References}

1. Torres M, Laguna-Barraza R, Dalmases M, et al. Male fertility is reduced by chronic intermittent hypoxia mimicking sleep apnea in mice. Sleep 2014;37:1757-65.

2. Ma L, Zhang J, Liu Y. Roles and Mechanisms of Obstructive Sleep Apnea-Hypopnea Syndrome and Chronic Intermittent Hypoxia in Atherosclerosis: Evidence and Prospective. Oxid Med Cell Longev 2016;2016:8215082.

3. Kiernan EA, Smith SM, Mitchell GS, et al. Mechanisms of microglial activation in models of inflammation and hypoxia: Implications for chronic intermittent hypoxia. J Physiol 2016;594:1563-77.

4. Sforza E, Roche F. Chronic intermittent hypoxia and obstructive sleep apnea: an experimental and clinical approach. Hypoxia (Auckl) 2016;4:99-108.
5. Gagnon K, Baril AA, Gagnon JF, et al. Cognitive impairment in obstructive sleep apnea. Pathol Biol (Paris) 2014;62:233-40.

6. Macey PM, Prasad JP, Ogren JA, et al. Sex-specific hippocampus volume changes in obstructive sleep apnea. Neuroimage Clin 2018;20:305-17.

7. Joo EY, Kim H, Suh S, et al. Hippocampal substructural vulnerability to sleep disturbance and cognitive impairment in patients with chronic primary insomnia: magnetic resonance imaging morphometry. Sleep 2014;37:1189-98.

8. Snyder B, Shell B, Cunningham JT, et al. Chronic intermittent hypoxia induces oxidative stress and inflammation in brain regions associated with early-stage neurodegeneration. Physiol Rep 2017;5:e13258.

9. Passali D, Corallo G, Yaremchuk S, et al. Oxidative stress in patients with obstructive sleep apnoea syndrome. Acta Otorhinolaryngol Ital 2015;35:420-5.

10. Lu W, Kang J, Hu K, et al. The role of the Nox4derived ROS-mediated RhoA/Rho kinase pathway in rat hypertension induced by chronic intermittent hypoxia. Sleep Breath 2017;21:667-77.

11. Yürüker V, Nazıroğlu M, Şenol N. Reduction in traumatic brain injury-induced oxidative stress, apoptosis, and calcium entry in rat hippocampus by melatonin: Possible involvement of TRPM2 channels. Metab Brain Dis 2015;30:223-31.

12. Yan D, An G, Kuo MT. C-Jun N-terminal kinase signalling pathway in response to cisplatin. J Cell Mol Med 2016;20:2013-9.

13. Karatug Kacar A, Bolkent S. Necrotic cell death occur via JNK pathway with the activity of transcription factor c-Jun by 4-MC in INS-1 cell line. J Cell Biochem 2018;119:2048-60.

14. Li C, Gao Y, Tian J, et al. Sophocarpine administration preserves myocardial function from ischemia-reperfusion in rats via NF-kappaB inactivation. J Ethnopharmacol 2011;135:620-5.

15. Lee MY, Wang Y, Mak JC, et al. Intermittent hypoxia induces NF-kappaB-dependent endothelial activation via adipocyte-derived mediators. Am J Physiol Cell Physiol 2016;310:C446-55.

16. Alawdi SH, El-Denshary ES, Safar MM, et al. Neuroprotective Effect of Nanodiamond in Alzheimer's Disease Rat Model: a Pivotal Role for Modulating NF-kappaB and STAT3 Signaling. Mol Neurobiol 2017;54:1906-18.

17. Reiter RJ, Calvo JR, Karbownik M, et al. Melatonin and its relation to the immune system and inflammation. Ann N Y Acad Sci 2000;917:376-86. 
18. Dwivedi DK, Kumar D, Kwatra M, et al. Voluntary alcohol consumption exacerbated high fat dietinduced cognitive deficits by NF-kappaB-calpain dependent apoptotic cell death in rat hippocampus: Ameliorative effect of melatonin. Biomed Pharmacother 2018;108:1393-403.

19. Tuzcu M, Baydas G. Effect of melatonin and vitamin E on diabetes-induced learning and memory impairment in rats. Eur J Pharmacol 2006;537:106-10.

20. Alcala M, Sanchez-Vera I, Sevillano J, et al. Vitamin E reduces adipose tissue fibrosis, inflammation, and oxidative stress and improves metabolic profile in obesity. Obesity (Silver Spring) 2015;23:1598-606.

21. Lee J, Rhee MH, Kim E, et al. BAY 11-7082 is a broadspectrum inhibitor with anti-inflammatory activity against multiple targets. Mediators Inflamm 2012;2012:416036.

22. Jiang W, Li M, He F, et al. Targeting the NLRP3 inflammasome to attenuate spinal cord injury in mice. J Neuroinflammation 2017;14:207.

23. Zheng B, Zhang S, Ying Y, et al. Administration of Dexmedetomidine inhibited NLRP3 inflammasome and microglial cell activities in hippocampus of traumatic brain injury rats. Biosci Rep 2018;38. doi: 10.1042/BSR20180892

24. Onishi $M$, Iinuma $M$, Tamura $Y$, et al. Learning deficits and suppression of the cell proliferation in the hippocampal dentate gyrus of offspring are attenuated by maternal chewing during prenatal stress. Neurosci Lett 2014;560:77-80.

25. Suzuki A, Iinuma M, Hayashi S, et al. Maternal chewing during prenatal stress ameliorates stress-induced hypomyelination, synaptic alterations, and learning impairment in mouse offspring. Brain Res 2016;1651:36-43.

26. Lee CH, Park JH, Ahn JH, et al. Effects of melatonin on cognitive impairment and hippocampal neuronal damage in a rat model of chronic cerebral hypoperfusion. Exp Ther Med 2016;11:2240-6.

27. Kiasalari Z, Khalili M, Shafiee S, et al. The effect of Vitamin E on learning and memory deficits in intrahippocampal kainate-induced temporal lobe epilepsy in rats. Indian J Pharmacol 2016;48:11-4.

28. Dong P, Zhao J, Li N, et al. Sevoflurane exaggerates cognitive decline in a rat model of chronic intermittent hypoxia by aggravating microglia-mediated neuroinflammation via downregulation of PPAR-gamma in the hippocampus. Behav Brain Res 2018;347:325-31.

29. Gao H, Han Z, Huang S, et al. Intermittent hypoxia caused cognitive dysfunction relate to miRNAs dysregulation in hippocampus. Behav Brain Res 2017;335:80-7.
30. Al-Qahtani JM, Abdel-Wahab BA, Abd El-Aziz SM. Long-term moderate dose exogenous erythropoietin treatment protects from intermittent hypoxia-induced spatial learning deficits and hippocampal oxidative stress in young rats. Neurochem Res 2014;39:161-71.

31. Varfolomeev E, Vucic D. Intracellular regulation of TNF activity in health and disease. Cytokine 2018;101:26-32.

32. Chen L, Ruan Y, Wang X, et al. BAY 11-7082, a nuclear factor-kappaB inhibitor, induces apoptosis and $\mathrm{S}$ phase arrest in gastric cancer cells. J Gastroenterol 2014;49:864-74.

33. Xu J, Qin X, Cai X, et al. Mitochondrial JNK activation triggers autophagy and apoptosis and aggravates myocardial injury following ischemia/reperfusion. Biochim Biophys Acta 2015;1852:262-70.

34. Sato M, Bagchi D, Tosaki A, et al. Grape seed proanthocyanidin reduces cardiomyocyte apoptosis by inhibiting ischemia/reperfusion-induced activation of JNK-1 and C-JUN. Free Radic Biol Med 2001;31:729-37.

35. Zeke A, Misheva M, Remenyi A, et al. JNK Signaling: Regulation and Functions Based on Complex ProteinProtein Partnerships. Microbiol Mol Biol Rev 2016;80:793-835.

36. Zerbini LF, de Vasconcellos JF, Czibere A, et al. JunDmediated repression of GADD45alpha and gamma regulates escape from cell death in prostate cancer. Cell Cycle 2011;10:2583-91.

37. Smith GB, Mocarski ES. Contribution of GADD45 family members to cell death suppression by cellular Bcl-xL and cytomegalovirus vMIA. J Virol 2005;79:14923-32.

38. Han J, Guo X, Tan W, et al. The expression of p-ATF2 involved in the chondeocytes apoptosis of an endemic osteoarthritis, Kashin-Beck disease. BMC Musculoskelet Disord 2013;14:209.

39. Pant I, Rao SG, Kondaiah P. Role of areca nut induced JNK/ATF2/Jun axis in the activation of TGF-beta pathway in precancerous Oral Submucous Fibrosis. Sci Rep 2016;6:34314.

40. Kielb SA, Ancoli-Israel S, Rebok GW, et al. Cognition in obstructive sleep apnea-hypopnea syndrome (OSAS): current clinical knowledge and the impact of treatment. Neuromolecular Med 2012;14:180-93.

Cite this article as: Liu F, Liu TW, Kang J. The role of NF$\kappa \mathrm{B}$-mediated JNK pathway in cognitive impairment in a rat model of sleep apnea. J Thorac Dis 2018;10(12):6921-6931. doi: 10.21037/jtd.2018.12.05 